\title{
EVALUACIÓN DEL USO DE ATMÓSFERA MODIFICADA EN FRAGARIA CHILOENSIS L. ECOTIPO BLANCO
}

\author{
EVALUATION OF THE USE OF MODIFIED ATMOSPHERE IN FRAGARIA \\ CHILOENSIS L. WHITE ECOTYPE
}

\author{
Nelson Loyola López ; ; Manuel Barrera Salas; Carlos Acuña Carrasco
}

\begin{abstract}
RESUMEN
En Chile se producen dos especies de frutillas, Fragaria chiloensis L. y Fragaria ananassa L. Duch. El uso de atmósfera modificada (AM) mediante una película plástica surge como alternativa que permitiría prolongar la postcosecha y conservación de las propiedades organolépticas de los frutos de Fragaria chiloensis L., altamente perecible. El presente estudio evaluó la efectividad de las bolsas de AM (FF 604 de 200 $\mu$ UPVC/70 $\mu$ LDPE) en la conservación de las propiedades sensoriales y en la prolongación de la vida de postcosecha de frutos de F. chiloensis L. producidos en Curepto, VII Región, siendo evaluados durante 11 días los tratamientos: $\left(\mathrm{T}_{1}\right)$ Fruta seleccionada, embalada a $\mathrm{T}^{\mathrm{o}}$ de campo $\left(18{ }^{\circ} \mathrm{C}\right)$ en bolsa AM, almacenada a $0{ }^{\circ} \mathrm{C}$ y $90 \% \mathrm{HR} ;\left(\mathrm{T}_{2}\right)$ Fruta seleccionada a $18{ }^{\circ} \mathrm{C}$, embalada y preenfriada hasta temperatura de pulpa $2{ }^{\circ} \mathrm{C}$, en bolsa $\mathrm{AM}$, almacenada a $0{ }^{\circ} \mathrm{C}$ con $90 \% \mathrm{HR}$; $\left(\mathrm{T}_{0}\right)$ Fruta seleccionada a $18{ }^{\circ} \mathrm{C}$, embalada sin bolsa $\mathrm{AM}$ y almacenada a $0{ }^{\circ} \mathrm{C}$ y $90 \% \mathrm{HR}$. Se evaluó la concentración de $\mathrm{CO}_{2}$ y $\mathrm{O}_{2}$, y los atributos sensoriales: sabor, aroma, firmeza, color y aceptación general. El diseño experimental fue completamente al azar, con cuatro repeticiones y la unidad experimental fueron $800 \mathrm{~g}$ de fruta provenientes de dos cestillos de $400 \mathrm{~g}$ cada uno. $\mathrm{T}_{1}$ y $\mathrm{T}_{2}$ con $\mathrm{AM}$ permitieron prescindir del preenfriado, sin que esto afectara significativamente las propiedades organolépticas de la fruta. La bolsa tipo $\mathrm{AM}$ en sus tratamientos $\mathrm{T}_{1}$ y $\mathrm{T}_{2}$ contribuyó, según los panelistas, a mantener el aroma y la firmeza de la fruta, no así respecto del color y sabor.
\end{abstract}

Palabras clave: Fragaria sp., atmósfera modificada, evaluación sensorial.

\begin{abstract}
The strawberry (Fragaria spp.) is the most common berry fruit consumed in the world, and in Chile two species are produced (Fragaria chiloensis L. and Fragaria ananassa L. Duch). Its main problem is the short shell life that shows in post-harvest. This hinders the commercialization and export. The use of modified atmosphere (MA) by means of a plastic film arises like an alternative that would allow to extend the post-harvest and the conservation of the sensorial properties of the fruits of Fragaria chiloensis $\mathrm{L}$. The present study had as an objective to evaluate the effectiveness of the bags of MA in the preservation of the sensorial properties and to increase the shell life of Fragaria chiloensis L. Fruits of Fragaria chiloensis L. were harvested in Curepto near the coast of the seventh region of Chile and then analyzed in the science lab of the Universidad Católica del Maule in Curicó. Two treatments were made with MA using plastic films (FF 604 de $200 \mu$ UPVC/70 $\mu$ LDPE), in the $T_{1}$ and $T_{2}$, besides a witness $T_{0}$. $\left(T_{1}\right)$ : Fruits were selected and processed at field temperature $\left(18^{\circ} \mathrm{C}\right)$ in $\mathrm{MA}$ bags, and then stored at $0^{\circ} \mathrm{C}$ and $90 \% \mathrm{RH} ;\left(\mathrm{T}_{2}\right)$ : Fruits were selected at $18^{\circ} \mathrm{C}$, processed and pre-cooled until pulp temperature of $2^{\circ} \mathrm{C}$, in $\mathrm{MA}$ bags, and stored at $0^{\circ} \mathrm{C}$ with $90 \%$ RH; $\left(T_{0}\right)$ : Fruits selected at $18^{\circ} \mathrm{C}$, processed without MA and stored at $0^{\circ} \mathrm{C}$ with $90 \% \mathrm{RH}$. The concentrations of carbon dioxide and oxygen were measured, and also the sensory attributes like flavor, color, texture and general acceptance all of this for a period of 11 days. $T_{1} y$ $T_{2}$, allowed to avoid using the pre-cooled in fruits without any significant change in the organoleptic properties they have. $T_{1}$ y $T_{2}$ also contributed, according to the panelist, to maintain the aroma and texture of the fruits, but not the color and flavor.
\end{abstract}

Key words: Fragaria sp., modified atmosphere, sensory evaluation.

1 Universidad Católica del Maule, Departamento de Ciencias Agrarias. E-mail: nloyola@ucm.cl Carmen 684, Casilla 7-D, Curicó.

Fecha de Recepción: 17 Noviembre 2006

Fecha de Aceptación: 05 Marzo 2007 


\section{INTRODUCCIÓN}

\section{ANTECEDENTES GENERALES}

Chile es reconocido como un país netamente productor de materias primas en distintos rubros, ya sea minería, forestal, agricultura, industria pesquera y acuicultura. En este sentido, en los últimos años todas estas grandes industrias han visto crecer su rubro, a diferencia de la pequeña y mediana agricultura que no sólo se ha estancado, sino más bien ha retrocedido (Vildósola y Riveros, 2003). Este hecho ha obligado a los pequeños y medianos agricultores a buscar nuevas alternativas productivas y además los insta a ser más eficientes y eficaces en sus labores agrícolas, lo que implica un buen manejo productivo y nuevas especies a cultivar.

En esta búsqueda de ¿qué hacer?, está la posibilidad de incursionar en productos nuevos y exóticos en Chile, tales como el cultivo de: Hongo Shitake, Caracoles, Avestruces, entre otros. Esto ocurre a pesar de que en Chile se encuentran especies y variedades endémicas que presentan un gran potencial comercial y que aún no han sido explotadas completamente (Hancok et al., 1999).

La frutilla (Fragaria ananassa L. Duch.) es hoy en día uno de los frutos menores más consumidos, pero esta es sólo una de las tantas especies existentes en el mundo (Villagrán 1994). Lo anterior se atribuye a sus agradables características organolépticas, su alto contenido de vitamina $\mathrm{C}$, cantidad de polifenoles y su contenido de ácido elágico (el cual actúa como agente anticancerígeno), ello junto a la versatilidad que presenta para ser utilizada y consumida en diferentes formas, sea en fresco, mermeladas, dulces, bebidas y jugos, entre otros. Además, se mencionan otros beneficios, como su alto contenido de antioxidantes, que retardan el envejecimiento, la prevención de infecciones urinarias y la disminución del contenido de azúcar en la sangre (Villagrán, 2001).

La frutilla (Fragaria chiloensis L.) ecotipo blanco es una especie nativa del sur de Chile que ha pasado desapercibida para el mundo durante el transcurso de los años (Gambardella et al., 2002).

Señalan Lavín y Maureira (2000) que en el año 1714 el capitán francés Amadée François Frezier, en su regreso a Francia, llevó desde Penco plantas femeninas portadoras de fruta de Fragaria chiloensis L., plantas que los aborígenes cultivaban desde antes de la llegada de los españoles. Algunos cruzamientos entre Fragaria chiloensis L. y F. virginiana Duch, siendo esta última frutilla roja originaria de
Norteamérica, fueron las que originaron híbridos con flores hermafroditas y descendencia indefinidamente fértil, lo que justificó su determinación como una nueva especie híbrida que se clasificó como Fragaria ananassa L. Duch (Latorre, 1994). Es así como $F$. chiloensis L. aportó el 50\% de su material genético, para crear la "frutilla moderna", con sus diferentes variedades y características (Lavín y Maureira, 2000; Lavín 2003).

Esta especie ya era cultivada por los mapuches antes de la llegada de los españoles, quienes la denominaban Kellen o Quellghen. El cultivo de ecotipo blanco permanece como tal en el predio entre 10 y 15 años, por su resistencia a plagas, a diferencia del ecotipo rojo, que sólo perdura de dos a tres años (Villagrán, 1994).

No existe registro exacto acerca de la superficie F. chiloensis en el país; sin embargo, algunos autores señalan a la VIII Región como la más importante a nivel nacional (Lavín y Maureira, 2000). Según Ortiz (2004), el ecotipo de frutilla blanca actualmente se encontraría desde la zona costera de Iloca hacia el sur de Concepción.

La falta de conocimiento técnico y la poca tecnología aplicada al manejo de postcosecha son algunos de los principales problemas que afectan a este cultivo, debido a la corta vida útil que presentan los frutos después de cosechados, lo que impide lograr un buena comercialización (Morales y González, 2000).

De aquí la gran importancia que tienen las nuevas tecnologías aplicadas al manejo de postcosecha de los productos hortofrutícolas. Una de estas alternativas es el uso de atmósferas modificadas (AM), concepto que se define como: Tecnología usada como complemento a la conservación a baja temperatura para extender la vida de postcosecha de distintos productos hortofrutícolas (Parry, 1997).

Esta técnica ha demostrado muy buenos resultados en otras especies como cerezas (Prunnus avium) y kiwis (Actinidia deliciosa), entre otros (Villagra, 2004).

\section{HIPÓTESIS}

Se propone que el uso de atmósfera modificada (AM) del tipo pasivo permitiría mejorar la conservación de las propiedades organolépticas, aumentando la vida de postcosecha de frutilla blanca F. chiloensis L. en condición de refrigeración para consumo fresco. 


\section{OBJETIVO GENERAL}

Evaluar y comparar la incidencia de la modificación de atmósferas sobre $F$. chiloensis L., ecotipo blanco y sus características sensoriales.

\section{OBJETIVOS ESPECÍFICOS}

- Comparar dos sistemas de almacenamiento: refrigerado versus refrigerado más atmósfera modificada (AM) sobre $F$. chiloensis L. durante un período de 11 días.

- Evaluar la incidencia de la temperatura de pulpa de $F$. chiloensis L. en la conservación de la fruta, al momento del sellado de la bolsa con atmósfera modificada.

- Evaluar el efecto que tiene el uso de bolsas de AM sobre la conservación de las propiedades organolépticas características de la especie.

\section{MATERIALES Y MÉTODOS}

El experimento se llevó a cabo en distintas etapas. La etapa de cosecha de los frutos se realizó en la costa de la VII Región, específicamente en la comuna de Curepto, localidad de Huelón, sector costero ubicado a $75 \mathrm{~km}$ de la provincia de Curicó. Según Santibáñez y Uribe (1993), Huelón posee un régimen térmico caracterizado por temperaturas que varían en promedio entre una máxima de enero $24{ }^{\circ} \mathrm{C}$ y una mínima en julio $6,3{ }^{\circ} \mathrm{C}$. El período libre de heladas es de 339 días, con un promedio de una helada por año. Registra anualmente 1.329 días-grado y 576 horas de frío. El régimen hídrico observa una pluviometría media anual de $708 \mathrm{~mm}$, presenta un déficit hídrico de $766 \mathrm{~mm}$ y un período seco de siete meses. Recibe la influencia marina que modera las temperaturas estivales e invernales.

En esta zona existe una agricultura de secano y la frutilla blanca se encuentra relegada a pequeñas huertas familiares de no más de $1 / 4$ de ha/familia. ${ }^{1}$ En el lugar existen también frutillares más grandes de Fragaria ananassa L. Duch., además de cultivos tradicionales como porotos, entre otros, siendo el fuerte de la comuna la explotación forestal. ${ }^{2}$

En dicho lugar se cosecharon y se seleccionaron los frutos en el mismo campo, de acuerdo a

1 López, 2004. Pequeño productor.

2 Basualto, 2004. Jefe de oficina de Prodesal. factores fitosanitarios. La selección se hizo evitando problemas de calidad y condición de la fruta, tales como pudriciones, machucones y heridas abiertas entre otros.

La cosecha de los frutos se realizó con un rango de (2 a 3) valor ordinal, de acuerdo a la medición subjetiva, que utilizan los pequeños agricultores y que consiste en medir la fuerza que opone el fruto al presionarlo entre el dedo índice y el pulgar (Cuadro 1).

Para el experimento se realizaron tres tratamientos. El tratamiento testigo $\mathrm{T}_{\mathrm{o}}$, cuya fruta se embaló a temperatura $18{ }^{\circ} \mathrm{C}$ y atmósfera ambiente con $0,03 \%$ de $\mathrm{CO}_{2}, 21 \%$ de $\mathrm{O}_{2}$ y sin envoltorio de AM (Cuadro 2).

Los tratamientos de atmósfera modificada (AM) $\mathrm{T}_{1}$ y $\mathrm{T}_{2}$ correspondieron al tipo de movimiento pasivo, lo que implica la modificación de $\mathrm{O}_{2}$ y $\mathrm{CO}_{2}$ mediante la tasa respiratoria de la fruta. A las muestras del tratamiento $T_{2}$ se realizaron prefríos, con el propósito de bajar la temperatura de pulpa de $18{ }^{\circ} \mathrm{C}$ provenientes del campo a $2{ }^{\circ} \mathrm{C}$ (Cuadro 3).

Cuadro 1

Parámetros de evaluación de la firmeza en frutillas (Fragaria spp)

\begin{tabular}{|l|c|}
\hline Textura & Valor Ordinal \\
\hline Dura & 4 \\
\hline Firme & 3 \\
\hline Blanda & 2 \\
\hline Muy blanda & 1 \\
\hline
\end{tabular}

Fuente: Folder, 1986.

Cuadro 2

Composición de la atmósfera de la tierra

\begin{tabular}{|l|l|c|}
\hline \multicolumn{2}{|c|}{ Elemento } & Concentración (\%) \\
\hline Nitrógeno & $\mathrm{N}$ & 78,03 \\
\hline Oxígeno & $\mathrm{O}$ & 20,99 \\
\hline Dióxido de carbono & $\mathrm{CO}_{2}$ & 0,03 \\
\hline Argón & $\mathrm{Ar}$ & 0,94 \\
\hline
\end{tabular}

Fuente: Parry, 1995. 


\section{Cuadro 3}

Tratamientos para 11 días de almacenamiento en AM y refrigerado de Fragaria chiloensis L. ecotipo blanco

\begin{tabular}{|l|l|}
\hline Tratamiento & \multicolumn{1}{|c|}{ Descripción } \\
\hline 1. Testigo $\mathrm{T}_{\mathrm{o}}$ & $\begin{array}{l}\text { Fruta seleccionada a } 18{ }^{\circ} \mathrm{C} \text {, embalada sin } \\
\text { bolsa AM y almacenada a } 0{ }^{\circ} \mathrm{C} \text { y } 90 \% \text { de } \\
\text { humedad relativa. }\end{array}$ \\
\hline 2. AM 1 $\mathrm{T}_{1}$ & $\begin{array}{l}\text { Fruta seleccionada, embalada a } \mathrm{T}^{\circ} \text { de campo } \\
\left(18{ }^{\circ} \mathrm{C}\right) \text { en bolsa AM y almacenada a } 0{ }^{\circ} \mathrm{C} \text { y } \\
90 \% \text { de humedad relativa. }\end{array}$ \\
\hline 3. AM 2 $\mathrm{T}_{2}$ & $\begin{array}{l}\text { Fruta seleccionada a } 18{ }^{\circ} \mathrm{C} \text {, embalada y Pre- } \\
\text { enfriada hasta } \mathrm{T}^{\circ} \text { de pulpa } 2{ }^{\circ} \mathrm{C} \text {. Sellado de } \\
\text { bolsa AM y almacenada a } 0 ~ \\
\text { humedad relativa. }\end{array}$ \\
\hline
\end{tabular}

Las bolsas de los tratamientos $\mathrm{T}_{1} \mathrm{y}_{2}$ representan una barrera al intercambio gaseoso dada su constitución de polímeros plásticos de permeabilidad selectiva. Las muestras de cada tratamiento fueron introducidas a una cámara de frío a $0{ }^{\circ} \mathrm{C}$ y $90 \%$ de humedad relativa por un período de 11 días. Los plásticos utilizados fueron donados por la empresa San Jorge Impresores para este ensayo y corresponden a bolsa FF 604 de $200 \mu$ UPVC/70 $\mu$ LDPE.

Para el buen uso de las bolsas AM, el sellado de ellas se realizó de la siguiente manera:

a) Al empacar el producto se tomó la precaución de levantar los bordes de la bolsa, para asegurar que el producto mantuviera el recubrimiento liso en la base.

b) Se juntaron los bordes del cuello de la bolsa, dejando salir el aire contenido dentro de ésta, retorciendo el cuello de la bolsa a modo de impedir que el aire vuelva a entrar.

c) Posteriormente se realizó el sellado del cuello de la bolsa, mediante el uso de una amarra.

d) Una vez sellada la bolsa, se puso el cuello a un costado, evitando dañar el envase (Anexo 1). Durante el transcurso del tiempo de almacenamiento fueron evaluados los parámetros siguientes: concentración de gases $\left(\mathrm{CO}_{2} \mathrm{y}\right.$ $\mathrm{O}_{2}$ ) con la ayuda de un analizador de gases MAPtest 4000 de origen inglés, cedido para este ensayo por la empresa privada San Jorge Impresores S.A. y también el peso neto de cada cestillo, con una balanza digital.
El empleo del equipo MAPtest 4000 consistió en la extracción de una muestra de la concentración de gases interna, de cada unidad muestral, mediante el uso de jeringas, procurando sellar cada perforación después de tomar las muestras. Dichas muestras de gases fueron posteriormente inyectadas al equipo, el cual arrojo un resultado exacto de la concentración de $\mathrm{CO}_{2}$ y $\mathrm{O}_{2}$ (Figura 1).

Posterior a la cosecha, durante la etapa de selección, basándose en parámetros de calidad y condición de la fruta y antes del embalaje, se realizó un análisis de evaluación sensorial de la fruta, el cual se define como una disciplina científica que permite medir, analizar e interpretar las características de un producto percibidas mediante los sentidos (Anzaldúa, 1994).

Las etapas antes mencionadas han sido resumidas en la Figura 2.

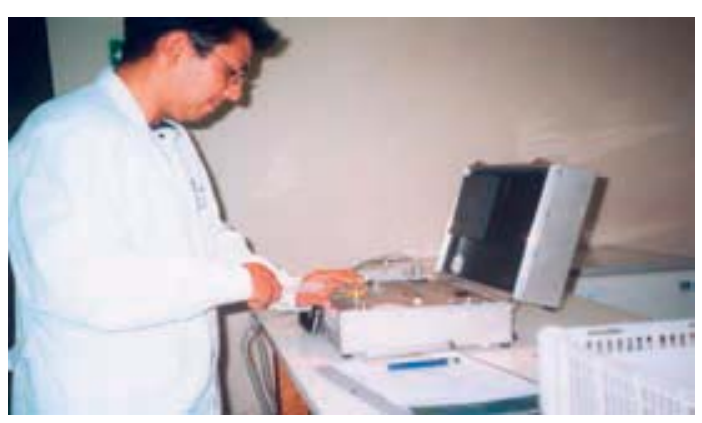

Figura 1. Uso del analizador de gases MAPtest 4000 durante el ensayo.

Anzaldúa (1994) señala que en la evaluación sensorial de alimentos se trabaja con panelistas, los que se seleccionan y entrenan con el fin de lograr la máxima veracidad, sensibilidad y reproducibilidad en los juicios que emitan, ya que de ellos dependerá en gran medida el éxito y la confiabilidad de los resultados. Mediante un entrenamiento adecuado, es posible obtener el mismo grado de seguridad que en un método instrumental, teniendo la ventaja de que la sensibilidad del test sensorial es mayor, debido a que los sentidos son capaces de pesquisar concentraciones menores.

El análisis sensorial está constituido por dos procesos definidos según su función: análisis sensorial y análisis estadístico. Mediante el primero, se obtienen las apreciaciones de los jueces a manera de datos que serán posteriormente transformados 


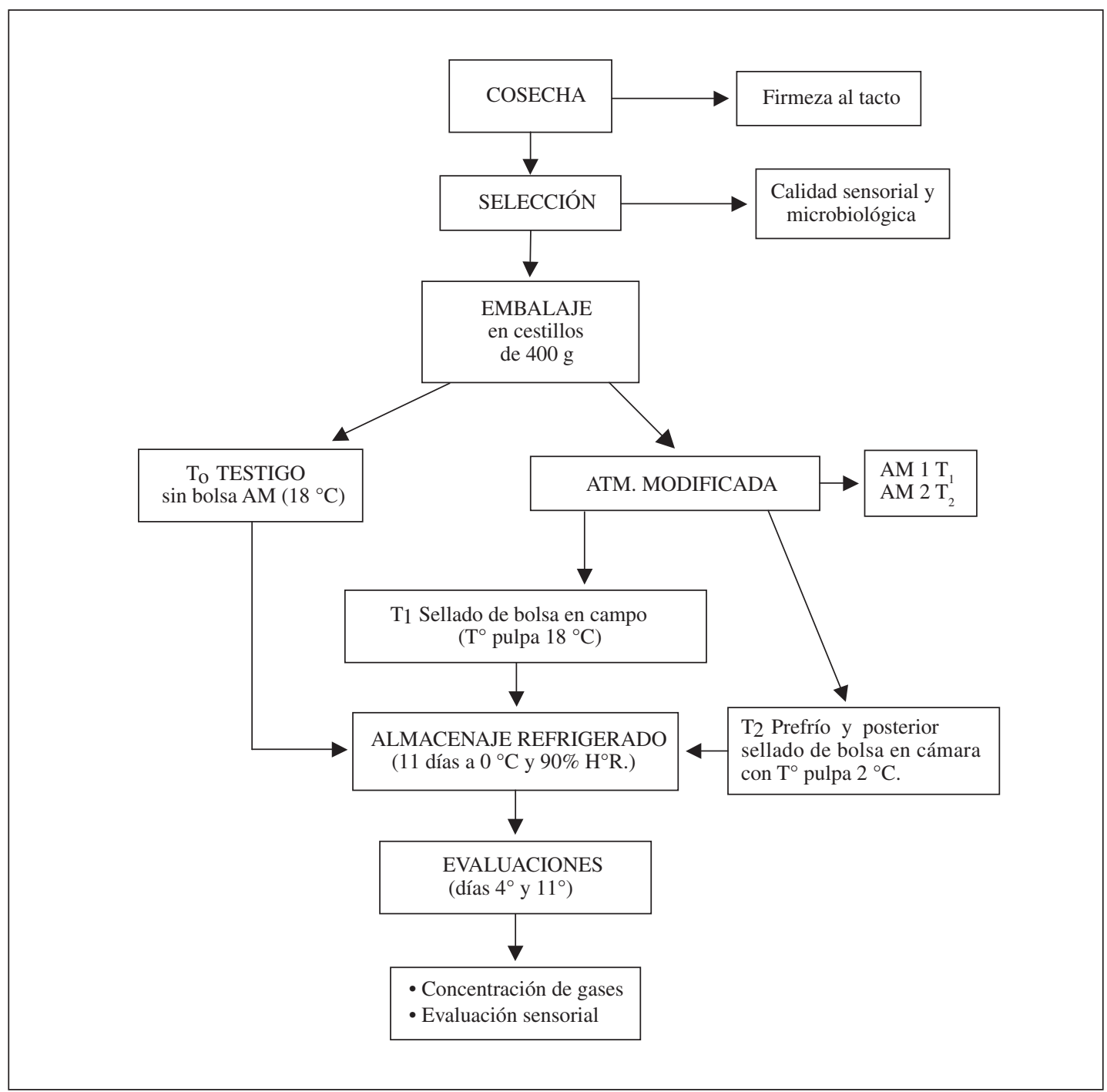

Figura 2. Línea de flujo tratamientos de Fragaria chiloensis L. ecotipo blanco, en refrigeración y almacenaje en AM.

y valorados por el segundo, dándoles con ello la objetividad deseada (Anzaldúa, 1994).

Para llevar a cabo el análisis sensorial se incluyó la participación de 13 panelistas entrenados y el uso de cartillas de evaluación sensorial. Esto permitió tener una apreciación real de los frutos y su potencial empleo para consumo humano, ya sea en estado fresco, o como base para su empleo en agroindustria.

Los panelistas entrenados recibieron una cartilla de evaluación sensorial y fueron instruidos en su uso, en la cual calificaron según el puntaje que cada uno estimó para cada pregunta.
La capacitación de los evaluadores se realizó en tres días, al inicio se les explicó su rol dentro del ensayo, los requisitos y deberes que debían cumplir. Se seleccionó principalmente a personas no fumadoras. A quienes sí se declararon fumadores se les explicó que no deberían fumar en los días en que se realizara la evaluación. En la eventualidad de que alguien fumara o se resfriara, debía informar al encargado del ensayo para ser reemplazado. Esto debido a que tanto el tabaco como un resfrío podrían afectar temporalmente la sensibilidad de los evaluadores, pudiendo inducir con ello a errores en la evaluación (Cuadro 4). 


\section{La capacitación se enfocó a cuatro parámetros:}

Color: Para esto se utilizaron cartulinas de colores A y B, además de frutilla natural, para establecer un parámetro de medición.

Sabor: Se utilizaron pocillos con distintos sabores: salado, dulce, amargo y picante, desconocidos por los evaluadores, con el objeto de establecer un parámetro de medición.

Textura: Se emplearon una bola de plasticina, una bola de acero, además de frutilla natural, con el objeto de establecer un parámetro de medición.

Aroma: Se utilizaron aromatizantes naturales, además de fruta, como asimismo canela para neutralizar el aroma entre cada medición.

Una vez realizada la evaluación sensorial y en base a los parámetros evaluados, los panelistas procedieron a calificar mediante una nota integradora de todas las propiedades medidas, constituyendo con ello una calificación final de aceptabilidad general para cada muestra (Cuadro 5).

\section{Cuadro 4}

Cartilla de evaluación sensorial para (Fragaria chiloensis L.) ecotipo blanco, almacenado a $0{ }^{\circ} \mathrm{C} \mathrm{y} 90 \%$ de humedad relativa por 11días

Instrucciones:

Usted ha sido seleccionado(a) para evaluar un nuevo producto que se lanzará al mercado próximamente, F. chiloensis L. ecotipo blanco, a calificar en una escala estructurada del 1 al 9.

\begin{tabular}{|l|c|c|}
\hline \multicolumn{1}{|c|}{ Nombre del juez: } & Fecha: & \\
\hline Clave de la muestra: & & \\
\hline ATRIBUTO & & \\
\hline SABOR & Rango & Puntaje \\
\hline a) Natural, deseable & 7 a 9 & \\
\hline b)Algo extraño a levemente indeseable & 4 a 6 & \\
\hline c) Fuertemente extraño, indeseable & 1 a 3 & \\
\hline INTENSIDAD DE AROMA & Rango & Puntaje \\
\hline a) Intenso & 7 a 9 & \\
\hline b) Moderado & 4 a 6 & \\
\hline c) Insípido & 1 a 3 & \\
\hline FIRMEZA: & Rango & Puntaje \\
\hline a) Firme & 7 a 9 & \\
\hline b) Algo firme & 4 a 6 & \\
\hline c) Blanda & 1 a 3 & \\
\hline COLOR: & Rango & Puntaje \\
\hline a) Rojo & 7 a 9 & \\
\hline b) Rosado & 4 a 6 & \\
\hline c) Blanco & 1 a 3 & \\
\hline
\end{tabular}

\section{Cuadro 5}

Cartilla de aceptabilidad general para

Fragaria chiloensis L. ecotipo blanco, almacenado a $0{ }^{\circ} \mathrm{C}$ y $90 \%$ de humedad relativa por 11 días

Instrucciones:

Usted ha sido seleccionado(a) para evaluar un nuevo producto que se lanzará al mercado próximamente $F$. chiloensis L. ecotipo blanco, a calificar en una escala estructurada del 1 al 9 .

\begin{tabular}{|l|c|c|}
\hline \multicolumn{1}{|c|}{ Aceptación general: } & Nota & Puntaje \\
\hline Excelente & 9 & \\
\hline Buena & 8 & \\
\hline Aceptable & 7 & \\
\hline Más que regular & 6 & \\
\hline Indiferente & 5 & \\
\hline Regular & 4 & \\
\hline Menos que regular & 3 & \\
\hline Mala & 2 & \\
\hline Inaceptable & 1 & \\
\hline
\end{tabular}

\section{EVALUACIONES}

Las evaluaciones físicas y sensoriales se llevaron a cabo en el Laboratorio de Ciencias de la Universidad Católica del Maule, Campus Nuestra Señora del Carmen Curicó.

Para la presente investigación se evaluaron parámetros tanto fisiológicos como: concentraciones de $\mathrm{CO}_{2}, \mathrm{O}_{2}$ y sensorial; aroma, sabor, color y textura, cuyos resultados se describen como sigue:

\section{ANÁLISIS FISIOLÓGICO}

Se realizaron tres evaluaciones los días 6, 10 y 17 de diciembre del 2004, con las cuales se pudo determinar la evolución de los dos gases antes mencionados, dentro del "filme" de AM, para cada uno de los tratamientos.

\section{ANÁLISIS SENSORIAL}

Se realizaron evaluaciones sensoriales, mediante la ayuda de un panel de evaluación, integrado por 13 personas entrenadas para dicho efecto, proceso anteriormente descrito.

Estas evaluaciones se llevaron a cabo los días 6, 10 y 17 de diciembre del 2004. 
Posteriormente, cada panelista efectuó una calificación de aceptación general, para cada uno de los tratamientos.

\section{DISEÑO EXPERIMENTAL}

El diseño experimental utilizado en este estudio fue completamente al azar, con cuatro repeticiones, y la unidad experimental fueron $800 \mathrm{~g}$ de frutos, provenientes de dos cestillos de $400 \mathrm{~g}$ cada uno.

En caso de existir diferencias estadísticas se efectuó una tabla de análisis de medias y pruebas de comparación múltiple según el test de Tukey con $\mathrm{p}>0,01$.

\section{RESULTADOS Y DISCUSIÓN}

En condiciones recreadas en el Laboratorio de Ciencias de la Universidad Católica del Maule en Curicó se logró cumplir la creación de atmósfera modificada (AM), mediante el uso de bolsas de permeabilidad selectiva. Dicha condición de AM resultó ser efectiva en la conservación de las propiedades organolépticas de Fragaria chiloensis L., ecotipo blanco, permitiendo con ello aumentar la vida útil de postcosecha de la especie. Estos resultados concuerdan con los mencionados por otros autores (Parry, 1997; Zoffoli, 1997), en relación al beneficio del uso de $\mathrm{AM}$ en distintos tipos de frutas y alimentos (Echeverría, 2003).

\section{RESULTADO DEL ANÁLISIS FISIOLÓGICO}

La medición en la concentración de gases del tratamiento $\mathrm{T}_{\mathrm{o}}$ se realizó sólo al inicio del ensayo, esto debido a que este último correspondió a un almacenamiento refrigerado en atmósfera normal.

El Cuadro 6 muestra la evolución del consumo de $\mathrm{O}_{2}$ y producción de $\mathrm{CO}_{2}$ de la fruta sometida al tratamiento $\mathrm{T}_{1}$ a diferentes temperaturas.

El tratamiento $\mathrm{T}_{1}$ presentó el mayor consumo de $\mathrm{O}_{2}$, a partir de la segunda fecha de evaluación, hecho propiciado principalmente por la temperatura de pulpa al momento de sellado de la bolsa $\mathrm{AM}, 18^{\circ} \mathrm{C}$. Esto último expresado gráficamente por una disminución de este gas y un aumento del $\mathrm{CO}_{2}$ (Figura 3).

\section{Cuadro 6}

Medición de concentración de gases $\left(\mathrm{O}_{2}\right.$ y $\left.\mathrm{CO}_{2}\right)$. Tratamiento 1 dentro de bolsas AM para Fragaria chiloensis L.

\begin{tabular}{|c|c|c|c|}
\hline Fecha & $\mathbf{O}_{\mathbf{2}}(\boldsymbol{\%})$ & $\mathbf{C O}_{\mathbf{2}}(\boldsymbol{\%})$ & $\begin{array}{c}\mathbf{T}^{\mathbf{o}} \mathbf{p u l p a} \\
\left({ }^{\circ} \mathbf{C}\right) .\end{array}$ \\
\hline 1) 06-12-2004 & 21,00 & 0,03 & 18 \\
\hline 2) 10-12-2004 & 16,80 & 2,70 & 2 \\
\hline 3) 17-12-2004 & 13,90 & 3,90 & 2 \\
\hline
\end{tabular}

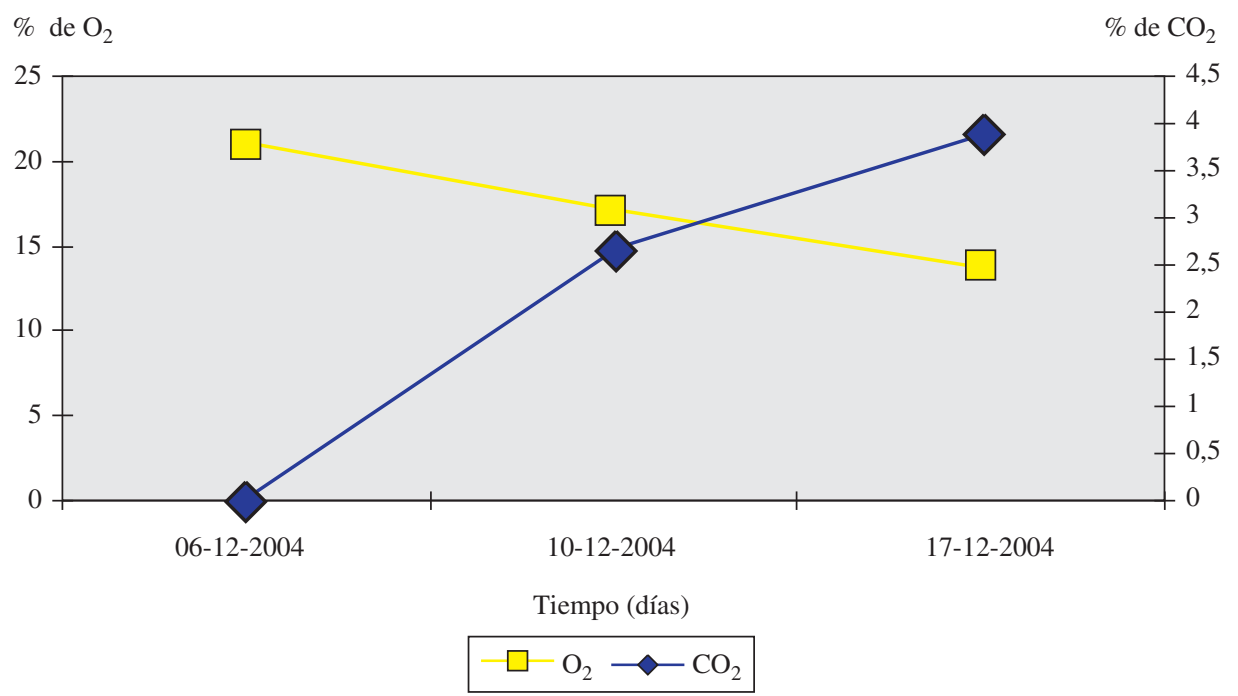

Figura 3. Evolución de la concentración de gases, dentro de la bolsa de AM tratamiento $\mathrm{T}_{1}$. 
El tratamiento $T_{2}$ presentó un intercambio gaseoso levemente menor al tratamiento $\mathrm{T}_{1}$, esto inducido por el prefrío al que fue sometida la fruta del tratamiento $\mathrm{T}_{2}$, previo al del sellado de la bolsa, reduciendo la temperatura de ésta a $2{ }^{\circ} \mathrm{C}$, lo que se acerca al óptimo de temperatura de almacenamiento para frutilla. Según Vial (1987), la temperatura óptima de almacenamiento de frutillas es $0{ }^{\circ} \mathrm{C}$, siendo su punto de congelación aproximadamente de $-1,1{ }^{\circ} \mathrm{C}$.

El Cuadro 7 relaciona la temperatura de pulpa de la fruta sometida al tratamiento $\mathrm{T}_{2}$ con los distintos niveles de concentración de $\mathrm{CO}_{2}$ y $\mathrm{O}_{2}$ dentro de la bolsa AM en cada una de las evaluaciones realizadas durante el desarrollo del ensayo.

A pesar de que no se midió directamente la tasa respiratoria de la fruta, estos valores son indicativos de una actividad respiratoria menor de la fruta dentro del envase $T_{2}$ en relación al tratamiento $T_{1}$.

Al realizar la segunda evaluación, el tratamiento $\mathrm{T}_{2}$ mostró un aumento en la producción de $\mathrm{CO}_{2} \mathrm{y}$ consumo de $\mathrm{O}_{2}$, hecho expresado gráficamente en la Figura 4.

Según Brody (1996), los cambios respiratorios de la fruta, provocados por la modificación de temperatura, pueden alterar las concentraciones de gases en equilibrio al interior del envase, así como el tiempo necesario para lograrlo.
De acuerdo al Cuadro 8 se puede observar que el análisis de varianza elaborado para la medición del nivel de oxígeno $\left(\mathrm{O}_{2}\right)$ indica que existió diferencia significativa entre los tratamientos.

\section{Cuadro 7}

Medición de concentración de gases

$\left(\mathrm{O}_{2}\right.$ y $\left.\mathrm{CO}_{2}\right)$. Tratamiento 2 dentro de bolsas AM para Fragaria chiloensis L.

\begin{tabular}{|c|c|c|c|}
\hline Fecha & $\mathbf{O}_{\mathbf{2}}(\boldsymbol{\%})$ & $\mathbf{C O}_{\mathbf{2}}(\boldsymbol{\%})$ & $\begin{array}{c}\mathbf{T}^{\mathbf{o}} \mathbf{p u l p a} \\
\left({ }^{\circ} \mathbf{C}\right)\end{array}$ \\
\hline $06-12-2004$ & 21,00 & 0,03 & 2,00 \\
\hline $10-12-2004$ & 18,80 & 1,80 & 2,00 \\
\hline $17-12-2004$ & 15,50 & 2,70 & 2,00 \\
\hline
\end{tabular}

\section{Cuadro 8}

Análisis de varianza para nivel de oxígeno ( $\left.\% \mathrm{O}_{2}\right)$ $(17 / 12 / 004)$

\begin{tabular}{|c|c|r|r|c|c|}
\hline FV. & GL & \multicolumn{1}{c|}{ SC } & CM & FC & FT \\
\hline Trat. & 2,00 & 111,28 & 55,64 & 3129,75 & 8,02 \\
\hline Error & 9,00 & 0,16 & 0,02 & & \\
\hline Total & 11,00 & 111,44 & & & \\
\hline
\end{tabular}

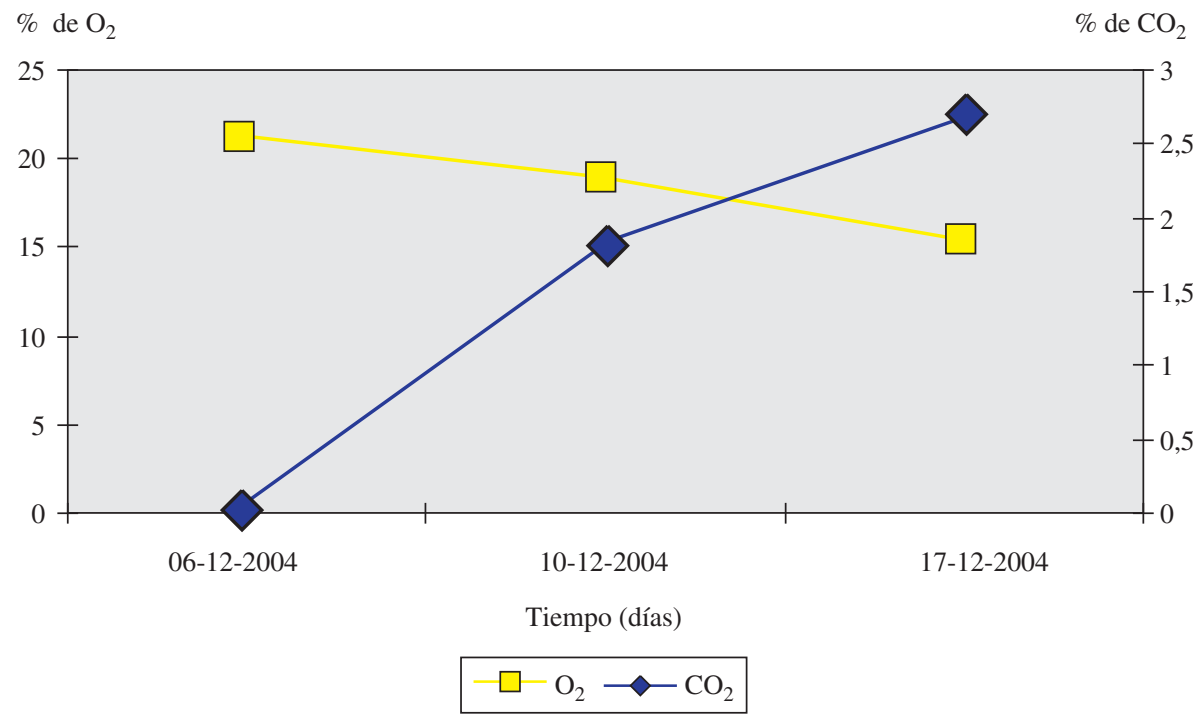

Figura 4. Evolución de la concentración de gases, dentro de la bolsa de (AM), tratamiento $T_{2}$. 


\section{Cuadro 9}

Comparaciones múltiples para nivel de oxígeno $\left(\% \mathrm{O}_{2}\right)$, según $($ Tukey $=0,01)$

\begin{tabular}{|l|c|c|c|c|}
\hline & & $\mathbf{T}_{\mathbf{o}}$ & $\mathbf{T}_{\mathbf{2}}$ & $\mathbf{T}_{\mathbf{1}}$ \\
\hline & & 21,00 & 15,52 & 13,57 \\
\hline $\mathrm{T}_{1}$ & 13,57 & 7,43 & 1,95 & - \\
\hline $\mathrm{T}_{2}$ & 15,52 & 5,48 & - & \\
\hline $\mathrm{T}_{\mathrm{o}}$ & 21,00 & - & & \\
\hline & & $\mathrm{a}$ & $\mathrm{b}$ & $\mathrm{c}$ \\
\hline
\end{tabular}

Al comparar los tratamientos entre sí, se determinó mediante análisis estadístico que todos aquellos resultaron distintos, es decir, que la evolución del oxígeno fue diferente en cada uno de los tratamientos evaluados (Cuadro 9).

La evolución del $\mathrm{O}_{2}$ fue distinta para cada tratamiento, hecho apreciable en la Figura 5, donde se muestran los tres tratamientos en cada fecha de evaluación del ensayo. Las letras ( $a$, b y c) indican distintos niveles de significancia entre tratamientos.

El tratamiento $\mathrm{T}_{1}$ presentó la mayor producción de $\mathrm{CO}_{2}$ en cada fecha de evaluación. Lo anterior ratifica lo señalado por Brody (1996), quien afirma que los productos hortofrutícolas presentan un consumo de $\mathrm{O}_{2}$ y producción de $\mathrm{CO}_{2}$ seis a ocho veces mayor a temperaturas cercanas a los $20^{\circ} \mathrm{C}$ que a $0{ }^{\circ} \mathrm{C}$.

El tratamiento $\mathrm{T}_{2}$ presentó una producción de $\mathrm{CO}_{2}$ significativamente menor respecto al tratamiento $\mathrm{T}_{1}$, observándose también la creación de una $\mathrm{AM}$ diferente a la creada en $\mathrm{T}_{1}$, a partir de la segunda fecha de evaluación.

De acuerdo al Cuadro 10 se puede observar que el análisis de varianza elaborado para la medición del nivel de dióxido de carbono $\left(\mathrm{CO}_{2}\right)$ indica que existió diferencia significativa entre los tratamientos.

Al comparar los tratamientos entre sí, se determinó que existió evidencia estadísticamente significativa que indica que todos los tratamientos son distintos, es decir, que la evolución del $\mathrm{CO}_{2}$ fue distinta en cada uno de los tratamientos (Cuadro 11).

Cuadro 10

Análisis de varianza para nivel de dióxido de carbono $\left(\% \mathrm{CO}_{2}\right),(17 / 12 / 004)$

\begin{tabular}{|c|c|c|c|c|c|}
\hline FV. & GL & SC & CM & FC & FT \\
\hline Trat. & 2,00 & 31,73 & 15,87 & 620,80 & 8,02 \\
\hline Error & 9,00 & 0,23 & 0,03 & & \\
\hline Total & 11,00 & 31,96 & & & \\
\hline
\end{tabular}

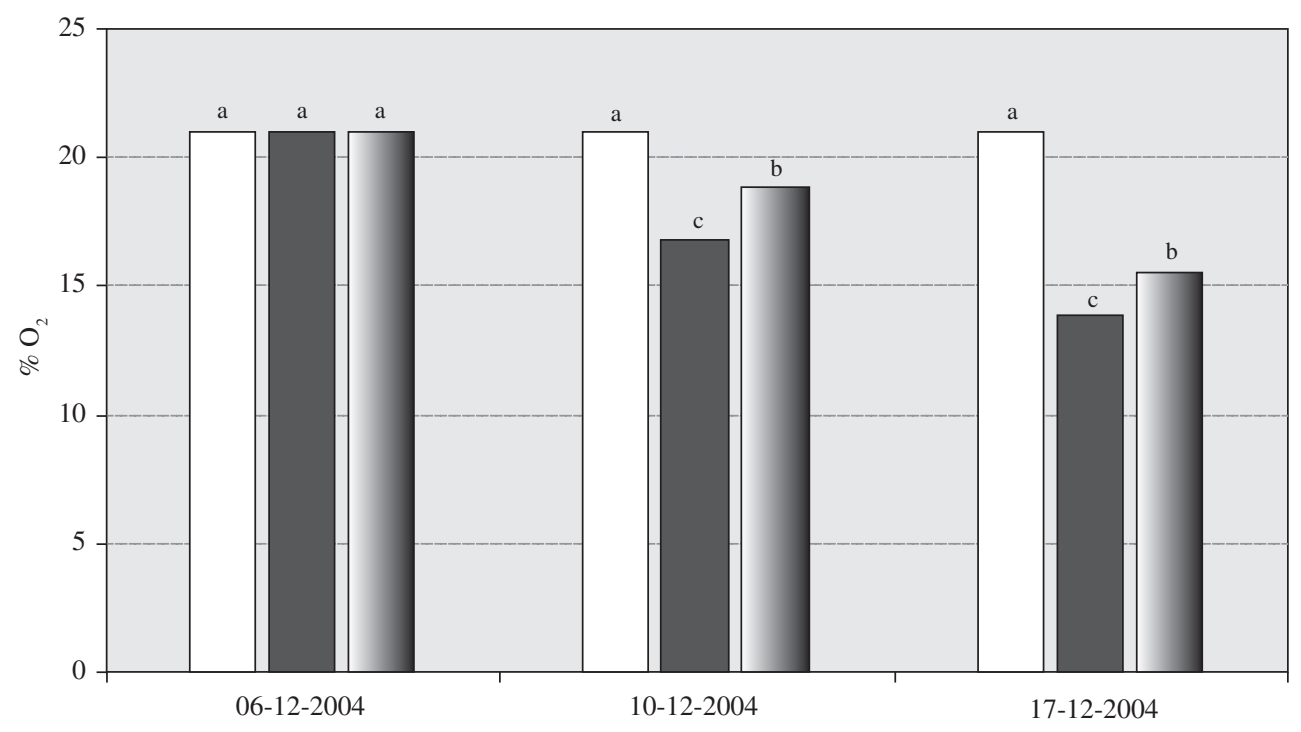

Almacenamiento (días) a $0{ }^{\circ} \mathrm{C}$ y $90 \% \mathrm{H}^{\circ} \mathrm{R}$.

$$
\square \text { Testigo } \square \text { Trat. } 1 \quad \square \text { Trat. } 2
$$

Figura 5. Evolución del oxígeno de cada tratamiento, en tres fechas de evaluación. 


\section{Cuadro 11}

Comparaciones múltiples, para nivel de dióxido de carbono $\left(\% \mathrm{CO}_{2}\right)$, según $($ Tukey $=\mathbf{0 , 0 1})$

\begin{tabular}{|c|c|c|c|c|}
\hline & & $\mathbf{T}_{\mathbf{1}}$ & $\mathbf{T}_{\mathbf{2}}$ & $\mathbf{T}_{\mathbf{0}}$ \\
\hline & & 3,90 & 2,67 & 0,03 \\
\hline $\mathrm{T}_{\mathrm{o}}$ & 0,03 & 3,87 & 2,64 & - \\
\hline $\mathrm{T}_{2}$ & 2,70 & 1,20 & - & \\
\hline $\mathrm{T}_{1}$ & 3,90 & - & & \\
\hline & & $\mathrm{a}$ & $\mathrm{b}$ & $\mathrm{c}$ \\
\hline
\end{tabular}

Evaluando la condición de AM, creada por los tratamientos $\mathrm{T}_{1} \mathrm{y} \mathrm{T}_{2}$, se pudo encontrar que la producción de $\mathrm{CO}_{2}$ fue mayor y más rápida significativamente diferente al $\mathrm{T}_{2}$ en el tratamiento $\mathrm{T}_{1}$ que fue sometido a una temperatura de almacenaje que en su etapa inicial fue de $2^{\circ} \mathrm{C}$, menor a lo observado en el tratamiento $\mathrm{T}_{1}\left(18^{\circ} \mathrm{C}\right)$.

La evolución del $\mathrm{CO}_{2}$ fue significativamente diferente para cada tratamiento.

El testigo $T_{0}$ no presentó variación alguna en todo el ensayo, en cambio el tratamiento $\mathrm{T}_{1}$ presentó una mayor producción de $\mathrm{CO}_{2}$, lo que indicaría una mayor tasa respiratoria de la fruta sometida a la condición térmica expuesta.

El tratamiento $\mathrm{T}_{2}$ presentó una menor producción de $\mathrm{CO}_{2}$, debido probablemente a una menor actividad metabólica exhibida por la fruta sometida a dicha condición (Figura 6).
De los resultados obtenidos en el ensayo se desprende que la concentración de gases dentro la bolsa de AM, es una consecuencia de la temperatura de pulpa de cada tratamiento, pudiendo afectar además el tiempo de vida útil en postcosecha de la fruta.

La barrera provocada por la bolsa AM permitió reducir el intercambio de gases entre el interior del envase y la atmósfera, logrando con ello disminuir la tasa respiratoria de la fruta, aumentando la vida de postcosecha en un $90 \%$.

\section{RESULTADO DE ANÁLISIS SENSORIAL}

$\mathrm{Al}$ momento del inicio del ensayo, el 6 de diciembre del 2004, se realizó un análisis de evaluación sensorial, tomando una muestra del universo total de la fruta utilizada para el ensayo, que posteriormente se distribuyó entre los tres tratamientos $\left(\mathrm{T}_{\mathrm{o}}, \mathrm{T}_{1} \mathrm{y} \mathrm{T}_{2}\right)$ con el propósito de establecer los parámetros que se utilizaron como base de comparación para los análisis posteriores de la fruta (Figura 7).

La evaluación sensorial hecha a la frutilla blanca al inicio del ensayo presentó, según el juicio de los penalistas, óptimo aroma y firmeza, pero regular sabor y color, obteniendo en la calificación general una nota de 6 , en la escala de 1 a 9 .

En dichas evaluaciones se midieron los atributos: aroma, color, firmeza y sabor, obteniendo las más altas calificaciones a juicio de los panelistas en los atributos de firmeza e intensidad de aroma, con una nota de 8 . El color no superó la nota 3 , normal para esta especie, y el sabor presentó una calificación con nota 6 .

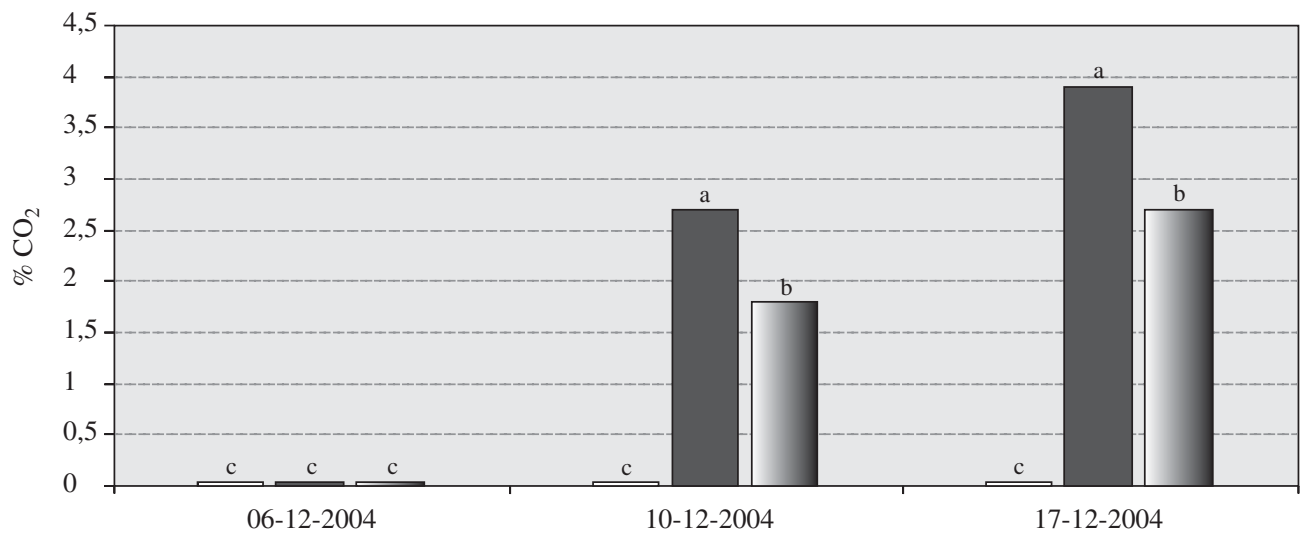

Almacenamiento (días) a $0{ }^{\circ} \mathrm{C}$ y $90 \% \mathrm{H}^{\circ} \mathrm{R}$.

Testigo $\square$ Trat. $1 \quad \square$ Trat. 2

Figura 6. Evolución de dióxido de carbono de cada tratamiento, en tres fechas de evaluación. 
Según Lavín y Maureira (2000), F. chiloensis L. corresponde a una fruta de gran sabor e intensidad de aroma, muy superior a su similar F. ananassa L. Duch. Estas afirmaciones contrastan con los resultados obtenidos en este ensayo, pudiendo deberse a que la fruta no se encontraba en su momento óptimo de madurez

La segunda evaluación se llevó a cabo el día 10 de diciembre del 2004. En dicha evaluación sensorial se observó que existió diferencia estadísticamente significativa en cuanto a la aceptabilidad general de la fruta entre el tratamiento testigo (To) y los tratamientos de atmósfera modificada $\mathrm{T}_{1} \mathrm{y} \mathrm{T}_{2}$.

Esta notoria diferencia se atribuye principalmente a la disminución de firmeza de la fruta, al igual que la intensidad del aroma. El sabor, según los panelistas, presentó un leve descenso con menor aceptación general, respecto del color, el que se mantuvo casi inalterado.

Al comparar los tratamientos $\mathrm{T}_{1}$ y $\mathrm{T}_{2}$ se determinó que no existió diferencia estadísticamente significativa en la aceptabilidad general entre ellos. Ambos tratamientos obtuvieron calificaciones similares en cada uno de los parámetros evaluados, además de presentar una mayor aceptabilidad general que el testigo. Lo anterior concuerda con lo señalado por Parry (1997) quien estableció que la reducción de los niveles de $\mathrm{O}_{2}$ y el enriquecimiento en $\mathrm{CO}_{2}$ pueden reducir la intensidad de la respiración del fruto, retrasar su maduración, disminuir la producción y la sensibilidad al etileno, retrasar la pérdida de textura, reducir la degradación de clorofila y el pardeamiento

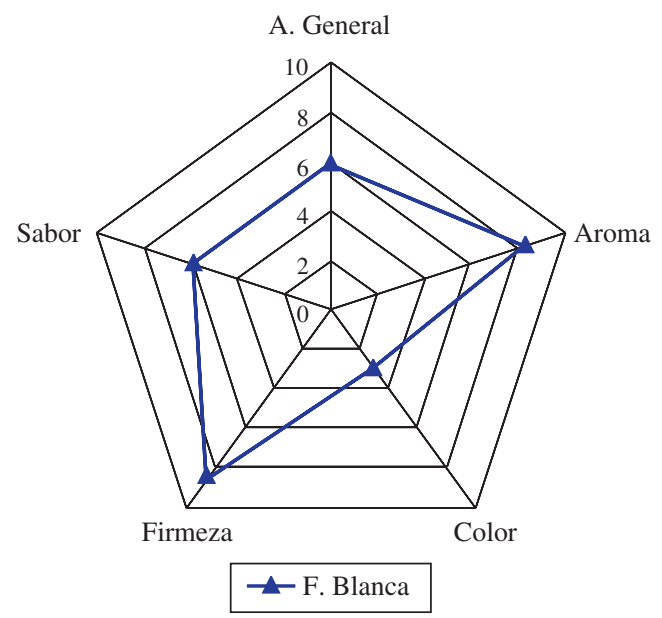

Figura 7. Evaluación sensorial y aceptación general de F. chiloensis L. al momento de inicio del ensayo (06-12-2004). enzimático, manteniendo las vitaminas y propiedades sensoriales de los frutos (Figura 8).

La diferencia significativa observada entre los tratamientos $\mathrm{T}_{1} \mathrm{y} \mathrm{T}_{2}$ respecto del testigo se atribuye al aporte positivo del envoltorio plástico (AM). Según Lavín y Maureira (2000), las principales propiedades sensoriales de Fragaria chiloensis L. son el sabor y la intensidad del aroma, ya que no se reconoce a esta especie por poseer un color muy atractivo.

Precisamente, uno de los aportes indirectos de la bolsa de atmósfera modificada es el de mantener y concentrar el aroma natural de la fruta, resaltando esta propiedad organoléptica, lo que fue percibido por los panelistas.

La tercera evaluación se llevó a cabo el día 17 de diciembre del 2004, obteniéndose como resultado que el tratamiento testigo presentó diferencias estadísticamente significativas en la aceptabilidad general en relación a los tratamientos de $\mathrm{AM} \mathrm{T}_{1} \mathrm{y} \mathrm{T}_{2}$.

Los panelistas advirtieron la presencia de olores desagradables y textura inconsistente en el tratamiento testigo, hecho que queda demostrado en la baja calificación general, la cual fue de 2,1 en la escala de 1 a 9. Esto último responde a lo señalado por Kirschbaum (1997), quien advierte de la alta perecibilidad de la frutilla en postcosecha, no superando los siete días en refrigeración, y contrasta con lo señalado por Lavín y Maureira (2000), quienes indican que es posible mantener frutos de Fragaria chiloensis L. en refrigeración por un período de 15 días, sin que esto implique la pérdida de sus propiedades sensoriales.

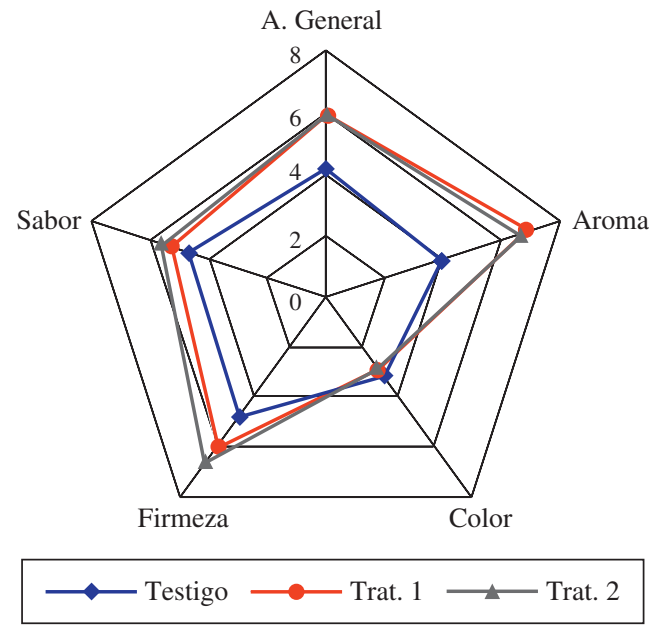

Figura 8. Evaluación sensorial y aceptación general de F. chiloensis L. en cada tratamiento (10-12-2004). 
En el presente ensayo, los tratamientos $\mathrm{T}_{1} \mathrm{y}_{2}$ lograron minimizar el grado de perecibilidad de la fruta, aumentando la vida de postcosecha a 11 días, manteniendo sus propiedades organolépticas.

Al comparar ambos tratamientos de AM, $\mathrm{T}_{1} \mathrm{y} \mathrm{T}_{2}$, se obtuvo que $\mathrm{T}_{2}$ presentó calificaciones ligeramente mayores que $\mathrm{T}_{1}$ en los parámetros de sabor y firmeza. No obstante, esto no influyó en la calificación de aceptabilidad general de la fruta, ya que no hubo diferencia estadísticamente significativa en la calificación de aceptabilidad general entre $\mathrm{T}_{1}$ y $\mathrm{T}_{2}$ (Figura 9).

No existió diferencia significativa en cuanto a aceptación general entre los tratamientos $\mathrm{T}_{1} \mathrm{y} \mathrm{T}_{2}$.

Los atributos sensoriales medidos no presentaron alteraciones negativas en la fruta sometida a los tratamientos $\mathrm{T}_{1}$ y $\mathrm{T}_{2}$, lo que se ratificó en la aceptación general evaluada por los panelistas; al respecto, por ello los tratamientos $\mathrm{T}_{1} \mathrm{y} \mathrm{T}_{2}$ demostraron ser mejores que el tratamiento $\mathrm{T}_{\mathrm{o}}$, el cual presentó calificaciones menores en cada uno de los atributos sensoriales evaluados.

Este resultado se ratifica con los datos entregados por la empresa San Jorge Impresores, la que señala que el uso de la bolsa de atmósfera modificada permitiría incrementar la vida útil de postcosecha de la frutilla, de manera similar a como ocurre con otras especies: como cerezas y kiwis, entre otros. ${ }^{3}$

Cabe señalar que el tratamiento $T_{1}$ presentó una mayor condensación de agua dentro del envase, pero, a pesar de ello, no se detectaron síntomas ni signos de enfermedades fungosas o de algún otro agente patógeno.

El principal aporte de las bolsas de AM es el de satisfacer las demandas del mercado, complementando los requerimientos de frío por parte de los frutos.

En el presente ensayo, a pesar de que existió diferencia estadísticamente significativa en el análisis fisiológico entre los tratamientos de AM $\mathrm{T}_{1} \mathrm{y} \mathrm{T}_{2}$, no existió diferencia significativa en la aceptabilidad general de la fruta, lo que se traduce en que el consumidor, representado por los panelistas, no sería capaz de diferenciar la frutilla proveniente de cualquiera de los tratamientos $\mathrm{T}_{1}$ y $\mathrm{T}_{2}$.

Dado que el tratamiento con AM $\mathrm{T}_{2}$ incluía prefrío y que este ultimo no contribuyó a una mayor aceptabilidad de la fruta por los panelistas, es factible asegurar que no es indispensable realizar el tratamiento de prefrío a la fruta para satisfacer las demandas de los consumidores, lo que implicaría

3 Rodríguez, 2004. Ingeniero Agrónomo, representante de San Jorge Impresores, S.A. un gran ahorro en la cadena de comercialización de la frutilla de exportación.

Como recomendación, y basándose en las condiciones de este ensayo, se sugiere realizar la cosecha de frutilla muy temprano por la mañana, evitando las horas de mayor temperatura, ya que este factor actúa en desmedro de la conservación de la fruta, acortando su vida útil. Lo anterior concuerda con lo señalado por autores como Kirschbaum (1997) quien señala que cada hora que la frutilla permanece sobre $27{ }^{\circ} \mathrm{C}$ su vida de postcosecha se reduce en un día. Por esto, el evitar las altas temperaturas, más el almacenamiento en la bolsa de atmósfera modificada pasiva, permitiría evitar un gasto adicional, derivado del uso de frío antes del embalaje, manteniendo la vida útil de postcosecha por un tiempo mínimo de 11 días.

Después de 11 días de almacenamiento refrigerado a $0{ }^{\circ} \mathrm{C}$ y $90 \%$ de humedad relativa, los frutos sometidos a envases de atmósfera modificada $\left(\mathrm{T}_{1} \mathrm{y}\right.$ $\mathrm{T}_{2}$ ) presentaron, según la evaluación de los panelistas, resultados sensoriales significativamente mejores en relación al tratamiento testigo $\mathrm{T}_{\mathrm{o}}$.

Relativo a la temperatura de pulpa de los frutos al momento del sellado del envase de $2{ }^{\circ} \mathrm{C}$ para $\mathrm{T}_{2}$ y $18{ }^{\circ} \mathrm{C}$ para $\mathrm{T}_{1}$, existió diferencia significativa entre los tratamientos $\mathrm{T}_{1} \mathrm{y} \mathrm{T}_{2}$ en la evolución de los gases $\left(\mathrm{CO}_{2} \mathrm{y} \mathrm{O}_{2}\right)$ al interior del los envases AM. A pesar de ello, esto no se tradujo en una diferencia significativa en la conservación de las propiedades organolépticas de la fruta entre los tratamientos $\mathrm{T}_{1} \mathrm{y} \mathrm{T}_{2}$.

La AM en sus tratamientos $\mathrm{T}_{1}$ y $\mathrm{T}_{2}$ contribuyó, según los panelistas, a mantener el aroma y la firmeza de la fruta, no así respecto del color y sabor (Figura 9).

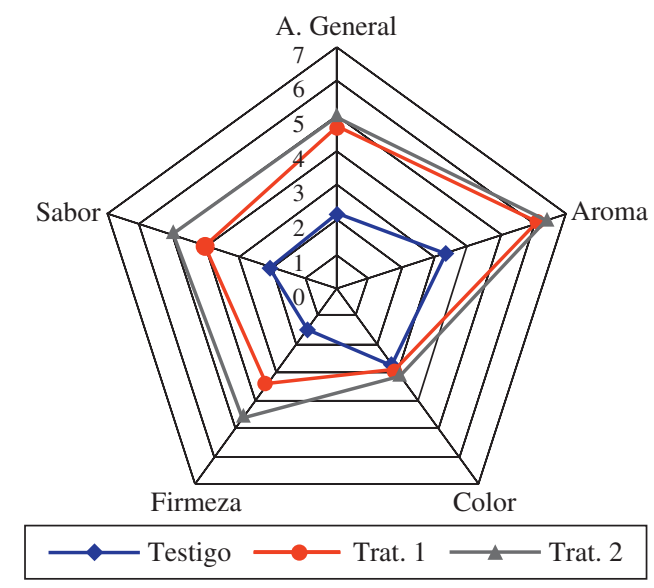

Figura 9. Evaluación sensorial y aceptación general de F. chiloensis L. en cada tratamiento (17-12-2004). 


\section{LITERATURA CITADA}

ANZALDÚA, A. 1994. La evaluación sensorial de los alimentos en la teoría y la práctica. Editorial Acribia, Zaragoza, España. 112 p.

BASUALTO, H. 2004. Jefe de oficina de Prodesal Curepto. Comunicación personal.

BRODY, A. 1996. Envasado de alimentos en atmósferas controladas, modificadas y al vacío. Editorial Acribia, S.A. España. 213 p.

ECHEVERRÍA, M. 2003. Uso de Atmósfera Modificada en Hortalizas. Tesis para optar al grado de Ingeniero de Ejecución Agrícola, Curicó, Chile. Universidad Católica del Maule. 61 p.

GAMBARDELLA, M., INFANTE, R., LÓPEZ-ARANDA, J.M., FAEDI, W. Y ROUDEIL, H. 2002. Collection of Wild and Cultivated Native Fragaria in Southern Chile. Acta Horticultura 567 (1): 61-63.

HANCOCK, J., LAVÍN, A. AND RETAMALES, J. 1999. Our Southern strawberry heritage: Fragaria chiloensis L. of Chile. HortScience 34: 818-816.

KIRSCHBAUM, D. 1997. Frutilla. Cosecha y manejo de postcosecha en Florida. Florida Agricultural State Servic. 40 p.

LATORRE, V. 1994. Proyecto Umag. Mejoramiento y calidad genética de plantas. Universidad de Magallanes. 65 p.

LAVÍN, A. Y MAUREIRA, M. 2000. La frutilla chilena de fruto blanco. Boletín INIA No 39 Cauquenes, Chile. 40 p.

LAVÍN, A. 2003. Ingeniero Agrónomo, INIA Quilamapu, Cauquenes VII región. Comunicación personal.

LÓPEZ. D. 2004. Pequeño productor de la zona de Huelón, comuna de Curepto, VII Región. Comunicación personal.

MORALES, G. Y GONZÁLEZ J. 2000. La Frutilla Nativa de Fruto Blanco: Mercado, Promoción y Análisis Económico. INIA - Quilamapu, Chillán. 55 p.
ORTIZ, J. 2004. El cultivo de la Frutilla (Fragaria chiloensis L.) ecotipo blanco. Tesis para optar al grado de Ingeniero Ejecución Agrícola, Curicó, Chile. Universidad Católica del Maule. 50 p.

PARRY, R. 1997. Envasado de los alimentos en atmósfera modificada. España, Madrid, Vicente ediciones. 330 p.

RODRÍGUEZ, J. 2004. Ingeniero Agrónomo, representante de San Jorge Impresores, S. A. Comunicación personal.

SANTIBÁÑEZ, F. Y URIBE, J.M. 1993. Atlas agroclimático de Chile. Regiones VI, VII, VIII y IX. Santiago de Chile. Universidad de Chile. Facultad de Ciencias Agrarias y Forestales. Blanco y negro. 99 p.

VIAL, R. 1987. Efecto del sistema de cosecha y atmósfera modificada en la calidad de postcosecha de frutilla (Fragaria ananassa Duch) cv. Tioga. Tesis para optar al grado de Ing. Agrónomo, Facultad de Agronomía, Universidad Católica de Chile. Santiago. 52 p.

VILLAGRÁN, V. 1994. El Cultivo de la Frutilla. FIA, Ministerio de Agricultura. Santiago de Chile. 112 p.

VILLAGRÁN, V. 2001. La Frutilla, Agenda del salitre Soquimich, $1515 \mathrm{p}$.

VILLAGRA,A. 2004. Atmósfera modificada en Cerezas (Prunnus avium) cv. Bing y en Kiwis (Actinidia deliciosa) cv. Hayward. Tesis para optar al grado de Ingeniero Ejecución Agrícola, Universidad Católica del Maule. Curicó. 65 p.

VILDÓSOLA, P. Y RIVEROS, J. 2003. Revista del campo, Economía: Bandas de precios. ¿Quién tiene la última palabra? Santiago de Chile. Año 28 (1405).

ZOFFOLI, J. P. 1997. Antecedentes para la optimización del sistema atmósfera modificada en cerezas. Revista Aconex. 56: 5-12. 